\title{
Cross-sectional association between objective cognitive performance and perceived age-related gains and losses in cognition
}

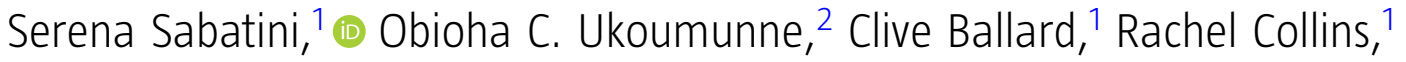 \\ Kaarin J. Anstey, ${ }^{3}$ Manfred Diehl, ${ }^{4}$ Allyson Brothers, ${ }^{4}$ Hans-Werner Wahl, ${ }^{5}$ \\ Anne Corbett, ${ }^{1}$ Adam Hampshire, ${ }^{6}$ Helen Brooker, ${ }^{1,7}$ and Linda Clare ${ }^{1,2}$ \\ ${ }^{1}$ Medical School, College of Medicine and Health, University of Exeter, Exeter, UK \\ ${ }^{2}$ Medical School, NIHR ARC South West Peninsula (PenARC), University of Exeter, Exeter, UK \\ ${ }^{3}$ Medical School, Ageing Futures Institute, University of New South Wales, Sydney, and Neuroscience Research Australia, Sydney, Australia \\ ${ }^{4}$ Department of Human Development and Family Studies, Colorado State University, Fort Collins, CO, USA \\ ${ }^{5}$ Medical School, Institute of Psychology, Heidelberg University, Heidelberg, Germany \\ ${ }^{6}$ Department of Brain Sciences, Imperial College London, London, UK \\ ${ }^{7}$ Ecog Pro Ltd., Bristol, UK
}

Objectives: Evidence linking subjective concerns about cognition with poorer objective cognitive performance is limited by reliance on unidimensional measures of self-perceptions of aging (SPA). We used the awareness of age-related change (AARC) construct to assess self-perception of both positive and negative age-related changes (AARC gains and losses). We tested whether AARC has greater utility in linking self-perceptions to objective cognition compared to well-established measures of self-perceptions of cognition and aging. We examined the associations of AARC with objective cognition, several psychological variables, and engagement in cognitive training.

Design: Cross-sectional observational study.

Participants: The sample comprised 6056 cognitively healthy participants (mean [SD] age $=66.0$ [7.0] years); divided into subgroups representing middle, early old, and advanced old age.

Measurements: We used an online cognitive battery and measures of global AARC, AARC specific to the cognitive domain, subjective cognitive change, attitudes toward own aging (ATOA), subjective age (SA), depression, anxiety, self-rated health (SRH).

Results: Scores on the AARC measures showed stronger associations with objective cognition compared to other measures of self-perceptions of cognition and aging. Higher AARC gains were associated with poorer cognition in middle and early old age. Higher AARC losses and poorer cognition were associated across all subgroups. Higher AARC losses were associated with greater depression and anxiety, more negative SPA, poorer SRH, but not with engagement in cognitive training.

Conclusions: Assessing both positive and negative self-perceptions of cognition and aging is important when linking self-perceptions to cognitive functioning. Objective cognition is one of the many variables - alongside psychological variables - related to perceived cognitive losses.

Key words: subjective cognitive complaints, subjective aging, attitudes toward own aging, AARC, self-perceptions of aging, depression, anxiety, perceived health

Global estimations suggest that 50 million people are living with dementia (World Health Organization,

Correspondence should be addressed to: Serena Sabatini, Medical School, College of Medicine and Health, Centre for Research in Ageing and Cognitive Health (REACH), University of Exeter, South Cloisters, St Luke's Campus, Exeter, EX12LU, UK. Phone: + 1392 726754; Fax: 01392 722972. Email: ss956@, exeter.ac.uk. Received 17 Nov 2020; revision requested 02 Jan 2021; revised version received 23 Feb 2021; accepted 27 Feb 2021. First published online 14 April 2021.
2020); a condition that creates a high social and economic burden. It is, therefore, important to identify individuals with poorer cognition as they may benefit the most from timely interventions aimed at preventing pathological cognitive decline. Self-perceptions of cognition and, more generally, self-perceptions of aging (SPA) may help to identify such individuals. Importantly, evidence on the 
association of self-reported cognitive limitations with objective cognition is inconclusive. Whereas, some studies found that self-reported cognitive limitations are correlated with poorer cognition and often precede pathological cognitive decline (Amariglio et al., 2012; Jessen et al., 2014), others found these associations to be either small or statistically irrelevant (Burmester et al., 2016; Crumley et al., 2014; Hertzog et al., 2018; Jonker et al., 2000).

These mixed results may be due to a lack of a recognized conceptualization of self-reported cognitive limitations (Rabin et al., 2015; Tandetnik et al., 2015). Among the many existing concepts of selfreported cognitive limitations, some (e.g. subjective memory decline) are used to refer to those people thought to have an early manifestation of dementia, whereas others (e.g. functional memory decline) are used to refer to memory complaints thought to be potentially reversible due to their associations with negative psychosocial factors (Blackburn et al., 2014). Moreover, some concepts (e.g. subjective cognitive decline) capture perceived decline in several domains (Slot et al., 2018), whereas others (e.g. subjective memory decline) focus solely on one domain (Hertzog et al., 2018). In addition, some studies assessed complaints about current cognitive difficulties, whereas others assessed perceived cognitive deterioration that occurred over time (Rabin et al., 2015). Another reason for the inconsistent evidence may be that individuals with specific psychological characteristics, such as depression and anxiety, can report cognitive decline that is not objectively measurable (Hill et al., 2016; Siebert et al., 2020). So far, most studies on the topic have focused on subjective memory decline (e.g. Hertzog et al., 2018), but individuals can experience a decline in several cognitive domains (Smart and Krawitz, 2015). It would, therefore, be sensible to use measures that cover subjective difficulties across several cognitive domains.

SPA, such as attitudes toward own aging (ATOA) and subjective age (SA), are also related to cognition. ATOA is commonly assessed with the ATOA scale (Lawton, 1975), which captures affective and cognitive components of self-related aging attitude. SA is frequently measured with a singleitem question asking individuals to report the age they feel they are (Barrett, 2003). Generally, more positive ATOA and/or feeling younger than one's chronological age are associated with better cognition (Seidler and Wolff, 2017), and consequently, with a lower risk of dementia (Siebert et al., 2018). This may be due to individuals with more positive SPA being more engaged in preventive behaviors and experiencing better mental and physical health (Bryant et al., 2012; Hess, 2006). These specific factors are protective against cognitive decline (Anstey, 2013).

Despite research supporting connections between more positive SPA and better cognition, existing evidence relies on unidimensional measures that treat positive and negative self-perceptions as two ends of the same spectrum (e.g. Barrett, 2003; Jorm and Jacomb, 1989; Lawton, 1975). These may provide an overly simplified picture. On one hand, with aging individuals often experience complex comorbidities, chronic health conditions, decline in some cognitive abilities (e.g. memory), decreased functional ability, and as a consequence of these losses, mild depressive, and anxiety symptoms (Palsson et al., 2001; Weyerer et al., 2013). On the other hand, aging also involves gains including valuable social relations, increased leisure time, and accumulated knowledge and life experience (Carstensen et al., 2011; Christensen, 2001; Steverink et al., 2001). Both positive and negative changes can impact on SPA. Moreover, many available measures (e.g. Barrett, 2003; Lawton, 1975) capture only global SPA, but not perceived age-related changes in cognition. Therefore, when linking self-perceptions to objective cognitive performance, it may be important to assess the coexistence of perceived age-related gains and losses in cognition.

The construct of awareness of age-related change (AARC) captures individuals' awareness that their behavior, performance, and/or life experiences have changed due to their increased age (Diehl and Wahl, 2010). AARC is the first concept assessing the coexistence of perceived gains and losses across five life domains: health and physical functioning; cognition; interpersonal relationships; sociocognitive and socio-emotional functioning; lifestyle. AARC is assessed via self-administered questionnaires; items capturing perceived gains and losses were derived from qualitative interviews and focus groups in which middle-aged and older individuals reported positive and negative aspects of aging (Brothers et al., 2019; Miche et al., 2014; Wahl et al., 2013). From both the 50-item (Brothers et al., 2019) and shorter 10-item (AARC-10 SF; Kaspar et al., 2019) versions of the AARC questionnaire, it is possible to obtain two global scores representing AARC gains and losses across life domains. In addition, the 50-item version of the questionnaire makes it possible to obtain 10 domain-specific scores; 1 for gains and 1 for losses in each of the 5 AARC life domains including the cognitive domain (AARC-50 cognitive functioning subscale; Brothers et al., 2019).

In contrast to some conceptualizations of subjective cognitive decline that attribute cognitive limitations to the development of brain pathology 
(Blackburn et al., 2014), AARC losses more broadly capture any self-reported cognitive limitation that individuals attribute to aging. Moreover, differently from unidimensional measures of self-reported cognitive limitations, AARC assumes that perceived cognitive losses coexist with cognitive gains. This is possible as AARC gains (in this paper referred to as social cognitive gains) and losses relate to different aspects of cognition: gains capture perceived improvements in knowledge, wisdom, and/or reflexivity, whereas losses capture perceived limitations in processing speed, memory, and/or mental capacity. Perceptions of social cognitive gains are quite independent from perceptions of cognitive losses (Sabatini et al., 2020b). As AARC captures selfperceptions of cognition across several cognitive domains (e.g. memory and processing speed), it makes it possible to link self-perceptions of cognition to a wide range of objectively assessed cognitive abilities.

Although the current study relies on crosssectional data, it adds important facets to previous research as it explores for the first time the relation between AARC and objective cognitive performance. First, as the measures used in this study make it possible to obtain both a global assessment of AARC and an assessment of AARC specific to the cognitive domain, this study tests whether an assessment of social cognitive gains and AARC losses specific to the cognitive domain is more strongly related to objective cognitive functioning, compared to a global assessment of AARC and to three well-established unidimensional measures of self-perceptions of cognition and aging (subjective cognitive changes, ATOA, SA). Second, given its large sample, this study robustly examines whether the associations of perceived social cognitive gains and cognitive losses with cognitive functioning vary among midlife, early old age, and advanced old age. Third, this study explores whether depression, anxiety, ATOA, SA, self-rated health (SRH), or level of engagement in computerized cognitive training explain variability in levels of AARC in cognition across age subgroups. Psychological variables, such as poor psychological health, are associated with negative SPA (Sabatini et al., 2020a; Siebert et al., 2020) and engagement in online cognitive training is linked to more positive self-perceptions of cognition (Sullivan et al., 2020).

We hypothesize that the assessment of perceived social cognitive gains and cognitive losses will be more strongly associated with cognitive functioning compared to a global assessment of AARC gains and losses, subjective cognitive change, ATOA, and SA. Second, we hypothesize that more AARC gains and fewer losses are associated with better cognitive performance across all age-based subgroups. Third, we hypothesize that more severe depression and/or anxiety, negative ATOA, an older SA, poorer SRH, or less engagement in computerized cognitive training are associated with fewer social cognitive gains and more AARC losses in cognition. We expect these associations to become stronger in older age as older individuals tend to be more accurate when self-evaluating their cognitive performance (Wang et al., 2004). Moreover, due to negative age stereotypes becoming increasingly salient with aging, it may be that when individuals in advanced old age experience cognitive decline, they are more likely to perceive themselves in a negative way compared to younger individuals (Meisner, 2012).

\section{Method}

\section{Study design and participants}

This study used secondary data collected from the ongoing PROTECT study (https://www. protectstudy.org.uk) in 2019 and 2020. Participants were 6056 cognitively healthy UK individuals aged 51.4-95.9 (age, $M=66.0$ years, $\mathrm{SD}=7.0$ years); of which $76.2 \%$ were women and 98.6\% were White. Among study participants, 3111 were middle aged (51-65 years); 2473 were in early old age (66-75 years), and 472 were in advanced old age ( $\geq 76$ years). Further information on study design and participants is provided in Supplementary text 1 .

\section{Measures}

\section{Indicators of self-perceptions of aging and cognition}

To assess perceived social cognitive gains and cognitive losses, we used the AARC-50 cognitive functioning subscale taken from the 50 -item version of the questionnaire (Brothers et al., 2019). Gains items capture social cognition and wisdom, whereas losses items capture perceived cognitive decline (memory, processing speed, etc.). To assess global perceptions of gains and losses across the five different AARC life domains, the AARC-10 SF (Kaspar et al., 2019) was used. For both the AARC-50 cognitive functioning subscale and the AARC-10 $\mathrm{SF}$, participants rate how much each of the 10 items (reported in Table 1) applies to them on a 5-point scale $(1=$ not at all; $5=$ very much). Scores can be obtained for gains and losses by summing the five items falling into the respective subscale. Higher scores indicate higher gains and losses (range: 5-25).

The Informant Questionnaire on Cognitive Decline in the Elderly short form was used 
Table 1. Items included in the AARC-50 cognitive functioning subscale and the AARC-10 SF

\begin{tabular}{|c|c|c|}
\hline \multicolumn{3}{|c|}{ AARC -50 cognitive functioning subscale items } \\
\hline \multirow{6}{*}{ AARC gains in cognition } & \multicolumn{2}{|c|}{ With my increasing age, I realize that... } \\
\hline & \multirow{2}{*}{\multicolumn{2}{|c|}{$\begin{array}{l}\text { I have more experience and knowledge to evaluate things and people. } \\
\text { I have more foresight. }\end{array}$}} \\
\hline & & \\
\hline & \multicolumn{2}{|c|}{ I gather more information before I make decisions. } \\
\hline & \multicolumn{2}{|c|}{ I have become wiser. } \\
\hline & \multicolumn{2}{|c|}{ I think things through more carefully. } \\
\hline \multirow[t]{5}{*}{ AARC losses in cognition } & \multicolumn{2}{|c|}{ My mental capacity is declining. } \\
\hline & \multicolumn{2}{|c|}{ I am slower in my thinking. } \\
\hline & \multicolumn{2}{|c|}{ I have a harder time concentrating. } \\
\hline & \multicolumn{2}{|c|}{ Learning new things takes more time and effort. } \\
\hline & \multicolumn{2}{|c|}{ I am more forgetful. } \\
\hline \multicolumn{3}{|l|}{ AARC-10 SF items } \\
\hline \multirow[t]{5}{*}{ AARC gains across life domains } & PHYS & I pay more attention to my health. \\
\hline & COG & I have more experience and knowledge to evaluate things and people. \\
\hline & INT & I appreciate relationship and people much more. \\
\hline & SCSE & I have a better sense of what is important for me. \\
\hline & LIFE & I have more freedom to live my days the way I want. \\
\hline \multirow[t]{5}{*}{ AARC losses across life domains } & PHYS & I have less energy. \\
\hline & COG & My mental capacity is declining. \\
\hline & INT & I feel more dependent on the help of others. \\
\hline & SCSE & I find it harder to motivate myself. \\
\hline & LIFE & I have to limit my activities. \\
\hline
\end{tabular}

PHY = Health and physical functioning; COG = Cognitive functioning; INT = Interpersonal relations; SCSE = Social-cognitive and social emotional functioning; LIFE $=$ Lifestyle and engagement.

(IQCODE-Self; Jorm and Jacomb, 1989) to assess subjective cognitive change over the last 10 years. It includes 16 items that are answered on a 5-point scale $(1=$ much improved; $5=$ much worse). The final score is the mean of the item scores; higher scores indicate subjective cognitive decline, whereas lower scores indicate subjective cognitive improvement.

In order to test whether objective cognition is more strongly associated with AARC gains and losses compared to other measures of SPA, we assessed ATOA and SA. We used the ATOA scale (taken from the Philadelphia Geriatric Center Morale Scale; Lawton, 1975) to assess ATOA. Lower scores indicate more negative ATOA, whereas higher scores indicate more positive ATOA (range: 0-5). To assess SA, participants were asked to write the age (in years) they feel most of the time (Barrett, 2003). A positive value indicates a younger SA, whereas a negative value indicates an older SA. To assess SRH, participants were asked to rate their health on a 4-point scale ( $4=$ Excellent; $1=$ Poor) (Ware and Sherbourne, 1992).

\section{Cognitive functioning}

To assess cognitive functioning, we used the PROTECT Cognitive Test Battery (Corbett et al., 2015), which was self-administered online and included four tests: (1) Self-Ordered Search (SOS) assesses spatial working memory (range: 0-20); (2) Grammatical Reasoning (GR) assesses verbal reasoning (range: from 0-no upper limit); (3) Paired Associate Learning (PAL) assesses visual episodic memory (range: 0-16); (4) Digit Span (DS) assesses verbal working memory $(0-20)$. For each test, a score is obtained by subtracting the number of errors from the number of correct answers; a higher score indicates better performance on the test. For GR, the score has no upper limit as the number of trials within the allocated time for the test varies depending on how rapidly participants respond during the test.

Through the PROTECT platform, participants have access to 12 online brain training games (validated by Owen et al., 2010) covering reasoning, problem-solving, mathematics, attention, and memory. The number of times participants played any brain training game between 2015 and 2019 was used as an indicator of the frequency of engagement in cognitive training. Further information on the assessment of cognitive functioning is presented in Supplementary text 2 .

\section{Mental health}

The Patient Health Questionnaire - 9 (Kroenke et al., 2001) was used to assess depression; higher scores indicate greater depression (range: 9-36). The Generalized Anxiety Disorder - 7 
(Spitzer et al., 2006) was used to assess anxiety; higher scores indicate more severe anxiety (range: 7-28).

Information on the reliability of study measures is presented in Supplementary text 3.

\section{Demographic information}

Demographic information included sex, employment status (employed; not employed), and education level (secondary education; postsecondary education; vocational qualification; undergraduate degree; postgraduate degree; doctorate).

\section{Data analysis}

For SA, a proportional discrepancy score was calculated by subtracting participants' SA from their chronological age and dividing this difference score by participants' chronological age. We fitted path analysis models to estimate the associations of participants' scores on the four objective cognitive tests (outcomes) with their scores on an assessment of perceived social cognitive gains and cognitive losses, a global assessment of AARC gains and losses, subjective cognitive change, ATOA, and SA. Path analysis made it possible to explore within one model the extent to which the coexistence of gains and losses (predictors) explains variability in the cognitive tests (outcomes). In the path analysis models, scores on objective cognitive tests were allowed to correlate. Sex, education, employment status, depression, anxiety, and frequency of cognitive training were included in the path analysis models as covariates. We treated depression, anxiety, and frequency of cognitive training as covariates as they likely impact on AARC and on objective cognition (Anstey, 2013). As the directions of all these associations have not been empirically investigated, we also tested a model excluding depression, anxiety, and frequency of cognitive training from covariates.

Partial coefficients of determination were not reported in the output from the path analysis models. However, as regression coefficients obtained with multiple linear regressions having gains and losses as predictors of scores on the cognitive tests led to similar results to those obtained with path analysis models, we reported results for the multiple regressions including information about the coefficients of determination in Supplementary Tables 3-5. To examine whether the strength of the associations of perceived social cognitive gains and cognitive losses (predictors) with cognitive performance varies among age subgroups, we estimated Pearson's $r$ correlation coefficients and three separate path analysis models for individuals in middle age, early old age, and advanced old age. The Comparative Fit Index (CFI), the Tucker-Lewis index (TLI), the Root Mean Square Error of Approximation (RMSEA), and the Standardized Root Mean Square Residual (SRMR) were used to assess model fit. Values considered acceptable were CFI and TLI > .90, RMSEA <.08 (90\% CI: 0; .08 ), and SRMR <.06 (Byrne, 2012).

To test whether more depression and/or anxiety, more negative ATOA, an older SA, poorer SRH, and frequency of cognitive training (predictors) are associated with fewer perceived social cognitive gains and more cognitive losses across age subgroups, we fitted simple and multiple regressions. Standardized coefficients are reported to quantify effects. Associations $\leq .09$ were considered negligible, .10-.29 small, .30-.49 moderate, and $\geq .50$ large (Cohen, 1988).

\section{Results}

\section{Descriptive data}

On average, participants perceived "a little bit" of social cognitive gains and cognitive losses; "quite a bit" of gains and "a little bit" of losses in the global assessment of AARC; reported subjective cognitive decline and mixed ATOA; felt $17 \%$ younger than their chronological age; and had minimal levels of depression and anxiety. Frequency of engagement in cognitive training varied greatly among participants. A high proportion of participants perceived their health as good $(54.6 \%)$ or excellent $(30.7 \%)$. Characteristics for the overall study sample, subsamples, and participants excluded from analyses are reported in Table 2.

\section{Indicators of self-perceptions of aging and cognition as predictors of cognitive functioning}

The associations of perceived social cognitive gains and cognitive losses, global levels of AARC gains and losses, subjective cognitive change, ATOA, and SA with cognitive performance are reported in Table 3 and Supplementary Table 1. Overall gains and losses in cognition and global levels of AARC gains and losses showed stronger associations with cognitive performance compared to subjective cognitive change, ATOA, and SA. However, the associations of cognitive performance with perceived social cognitive gains and cognitive losses and global levels of AARC gains and losses were either small or negligible. The global assessment of AARC gains and losses explained slightly more variance in cognitive functioning than the assessment of perceived social cognitive gains and cognitive losses. 
Table 2. Descriptive statistics of demographic variables and main study variables for the study sample, study subsamples, and participants not included in the study

\begin{tabular}{|c|c|c|c|c|c|}
\hline VARIABLES & $\begin{array}{l}\text { PARTICIPANTS NOT } \\
\text { INCLUDED IN THE } \\
\text { STUDY }(n=8826)\end{array}$ & $\begin{array}{l}\text { STUDY SAMPLE } \\
\quad(n=6056)\end{array}$ & $\begin{array}{l}\text { PARTICIPANTS IN } \\
\text { MIDDLE AGE } \\
(n=3111)\end{array}$ & $\begin{array}{c}\text { PARTICIPANTS } \\
\text { IN EARLY OLD AGE } \\
\quad(n=2473)\end{array}$ & $\begin{array}{l}\text { PARTICIPANTS } \\
\text { IN ADVANCED } \\
\text { OLD AGE } \\
(n=472)\end{array}$ \\
\hline Age (years), Mean (SD; range) & $65.05(7.23 ; 50.21,103.49)$ & $66.01(6.98 ; 51.38,95.93)$ & $60.48(3.53 ; 51,65)$ & $70.31(2.73 ; 66,75)$ & $\begin{array}{c}79.88(3.52 \\
76,95)\end{array}$ \\
\hline Sex (Women \%) & $6532(75.1)$ & $4615(76.2)$ & $2532(81.4)$ & $1789(72.34)$ & $294(62.3)$ \\
\hline \multicolumn{6}{|l|}{ Education level (\%) } \\
\hline Secondary & $1391(16.0)$ & $812(13.4)$ & $336(10.8)$ & $386(15.6)$ & $90(19.1)$ \\
\hline Postsecondary & $1048(12.0)$ & $682(11.3)$ & $355(11.4)$ & $269(10.9)$ & $58(12.3)$ \\
\hline Vocational qualification & $1763(20.3)$ & 1209. (20.0) & $596(19.2)$ & $519(20.6)$ & $103(21.8)$ \\
\hline Undergraduate degree & $2780(32.0)$ & $2062(34.1)$ & 1151. (37.0) & $768(31.1)$ & $143(30.3)$ \\
\hline Postgraduate degree & $1459(16.8)$ & $1506(17.4)$ & $580(18.6)$ & $431(17.4)$ & $45(9.5)$ \\
\hline Doctorate & $260(3.0)$ & $235(3.9)$ & $93(3.0)$ & $109(4.4)$ & $33(7.0)$ \\
\hline Current employment (Employed \%) & $4233(50.3)$ & $4233(50.3)$ & $2046(68.8)$ & $418(17.0)$ & $32(6.8)$ \\
\hline AARC gains in cognition, Mean (SD) & Not applicable & Not applicable & $13.88(4.38)$ & $13.26(4.34)$ & $13.11(4.27)$ \\
\hline AARC losses in cognition, Mean (SD) & Not applicable & Not applicable & $9.68(3.58)$ & $10.30(3.42)$ & $11.83(3.99)$ \\
\hline AARC gains across life domains, Mean (SD) & Not applicable & Not applicable & $17.77(3.97)$ & $17.56(3.88)$ & $17.27(3.78)$ \\
\hline AARC losses across life domains, Mean (SD) & Not applicable & Not applicable & $9.28(3.11)$ & $10.07(3.05)$ & $11.97(3.60)$ \\
\hline Self-ordered search, Mean (SD) & Not applicable & Not applicable & $8.12(2.52)$ & $7.50(2.51)$ & $6.71(2.49)$ \\
\hline Paired associate learning, Mean (SD) & Not applicable & Not applicable & $4.87(.91)$ & $4.67(.90)$ & $4.31(.89)$ \\
\hline Grammatical reasoning, Mean (SD) & Not applicable & Not applicable & $39.76(10.35)$ & $36.56(9.56)$ & $31.78(9.33)$ \\
\hline Digit span, Mean (SD) & Not applicable & Not applicable & $7.76(1.46)$ & $7.57(1.52)$ & $7.17(1.52)$ \\
\hline Depression, Mean (SD) & $11.81(3.41)$ & $11.81(3.41)$ & $11.71(3.14)$ & $11.07(2.55)$ & $11.17(2.62)$ \\
\hline Anxiety, Mean (SD) & $8.67(2.79)$ & $8.67(2.79)$ & $8.68(2.59)$ & $8.16(2.23)$ & $8.00(1.96)$ \\
\hline \multicolumn{6}{|l|}{ Self-rated health (\%) } \\
\hline Poor & $89(2.7)$ & $89(2.7)$ & $65(2.1)$ & $49(2.0)$ & $6(1.3)$ \\
\hline Fair & $453(13.5)$ & $453(13.5)$ & 396. (12.8) & $305(12.4)$ & 64. (13.6) \\
\hline Good & $1774(53.0)$ & $1774(53.0)$ & $1663(53.5)$ & 1377. (55.8) & $262(55.6)$ \\
\hline Excellent & $1029(30.8)$ & $1029(30.8)$ & $982(31.6)$ & $737(29.9)$ & $139(29.5)$ \\
\hline Subjective cognitive change, Mean (SD) & Not applicable & Not applicable & $3.08(.22)$ & $3.09(.21)$ & $3.13(.26)$ \\
\hline Attitudes toward own aging, Mean (SD) & Not applicable & Not applicable & $2.61(.77)$ & $2.64(.79)$ & $2.59(.93)$ \\
\hline Subjective age, Mean (SD) & Not applicable & Not applicable & $.17(.15)$ & $.17(.14)$ & $.17(.15)$ \\
\hline $\begin{array}{l}\text { Frequency of cognitive training; Mean } \\
\quad(\mathrm{SD} ; \text { range })\end{array}$ & Not applicable & Not applicable & $\begin{array}{l}307.11(759.60 \\
0,11602)\end{array}$ & $\begin{array}{l}474.51(1125.03 \\
0,13711)\end{array}$ & $\begin{array}{c}617.98(1446.27 \\
0,15,942)\end{array}$ \\
\hline
\end{tabular}


Table 3. Path analysis model exploring AARC gains and losses in cognition, AARC gains and losses across life domains, subjective cognitive change, ATOA, and SA as predictors of cognition in the overall study sample while controlling for sex, education, employment status, depression, anxiety, and frequency of cognitive training

STANDARDized COEFFICIENT $\beta(95 \% \mathrm{CI}) ;$-VAlUe

\begin{tabular}{|c|c|c|c|c|}
\hline PREDICTORS & SELF-ORDERED SEARCH & GRAMMATICAL REASONING & PAIRED ASSOCIATE LEARNING & DIGIT SPAN \\
\hline $\begin{array}{l}\text { AARC gains in cognition } \\
\text { AARC losses in cognition } \\
\text { RMSEA }(90 \% \mathrm{CI}): .07(.05, .09)\end{array}$ & $\begin{array}{l}-.06(-.09,-.04) ;<.001 \\
-.05(-.08,-.03) ;<.001 \\
\text { I: .99; TLI: .53; SRMR: .01; }\end{array}$ & $\begin{array}{l}-.09(-.11,-.07) ;<.001 \\
-.12(-.14,-.09) ;<.001 \\
19 \%\end{array}$ & $\begin{array}{l}-.02(-.05, .00) ; .083 \\
-.08(-.11,-.06) ;<.001\end{array}$ & $\begin{array}{l}-.04(-.07,-.02) ; .001 \\
-.06(-.09,-.04) ; .001\end{array}$ \\
\hline $\begin{array}{l}\text { AARC gains across life domains } \\
\text { AARC losses across life domains } \\
\text { RMSEA }(90 \% \mathrm{CI}): .06(.04, .09)\end{array}$ & $\begin{array}{l}-.04(-.07,-.02) ; .002 \\
-.07(-.10,-.04) ;<.001 \\
\text { I: .99; TLI: .62; SRMR: .01; }\end{array}$ & $\begin{array}{l}-.04(-.06,-.01) ; .002 \\
-.12(-.14,-.09) ;<.001 \\
21 \%\end{array}$ & $\begin{array}{l}-.02(-.04, .01) ; .160 \\
-.08(-.11,-.05) ;<.001\end{array}$ & $\begin{array}{l}-.01(-.03, .02) ; .648 \\
-.09(-.11,-.06) ;<.00\end{array}$ \\
\hline $\begin{array}{l}\text { Subjective cognitive change } \\
\text { RMSEA }(90 \% \mathrm{CI}): .00(.00, .04)\end{array}$ & $\begin{array}{l}.02 \text { (-.01, .05); .129 } \\
\text { I: } 1.0 \text {; TLI: } 1.0 \text {; SRMR: .00; }\end{array}$ & $\begin{array}{l}-.00(-.03, .02) ; .770 \\
12 \%\end{array}$ & $-.03(-.06,-.00) ; .045$ & $-.01(-.04, .02) ; .447$ \\
\hline Attitudes toward own aging & $.01(-.01, .04) ; .409$ & $.01(-.02, .03) ; .519$ & $-.00(-.03, .03) ; .996$ & $.00(-.03, .03) ; .960$ \\
\hline
\end{tabular}

RMSEA (90\% CI): .00 (.00, .00); CFI: 1.0; TLI: 1.0; SRMR: .00; R²: $11 \%$
Subjective age
$.01(-.02, .03) ; .665$
$.02(-.00, .05) ; .070$
$.04(.01, .06) ; .004$
$.03(.00, .05) ; .030$

RMSEA (90\% CI): .00 (.00, .00); CFI: 1.0; TLI: 1.0; SRMR: .00; R²: $9 \%$

RMSEA = Root mean square error of approximation; CFI = Comparative fit index; TLI = Tucker-Lewis index; SRMR = Standard root mean square residual; $\mathrm{R}^{2}=\mathrm{R}$-squared/coefficient of determination. 


\section{Associations of perceived social cognitive gains and cognitive losses with cognitive performance across age subgroups}

Estimates for correlations, path analysis models, and multiple linear regressions exploring the associations of perceived social cognitive gains and cognitive losses with scores on cognitive tasks across age subgroups are reported in Table 4 and Supplementary Tables $2-5$. Overall, higher social cognitive gains were associated with poorer scores on cognitive tests among participants in middle age and early old age. Although higher social cognitive gains were also related to poorer scores on cognitive tasks in advanced old age; these associations were not statistically significant. Higher AARC losses in cognition were associated with poorer performance on most cognitive tasks, especially on GR. These associations were consistent across all age subgroups but strongest in size in early old and advanced old age. For both perceived social cognitive gains and cognitive losses associations with scores on the cognitive tasks were either negligible or small; even though associations were slightly stronger in size for AARC losses.

\section{Associations of psychological variables and frequency of cognitive training with perceived social cognitive gains and cognitive losses across age subgroups}

Fewer social cognitive gains were associated with a younger SA in early old age, whereas the associations of social cognitive gains with depression, anxiety, ATOA, and SRH were either negligible or nonsignificant; see Table 5. Among participants in middle and early old age, more severe depression and anxiety, more negative ATOA, an older SA, and poorer SRH were associated with more AARC losses in cognition. Among participants in advanced old age, more severe depression and anxiety, an older SA, and poorer SRH showed small to moderate associations with more AARC losses in cognition (see Table 5). Higher engagement in computerized cognitive training was associated with higher social cognitive gains in middle age only and was not associated with AARC losses in any subgroup.

\section{Discussion}

This was the first study to examine whether the AARC-50 cognitive functioning subscale has greater utility in linking SPA to objective cognition compared to the AARC-10 SF and other well-established measures of self-perceptions of cognition and aging. This study was also the first to explore whether the coexistence of perceived social cognitive gains and cognitive losses is associated with objective cognitive functioning, a range of psychological variables, or frequency of engagement in cognitive training. Compared to unidimensional measures of SPA, measures capturing the coexistence of positive and negative age-related changes were more strongly associated with cognitive performance. However, the global assessment of AARC - encompassing perceptions of age-related changes across several life domains - is more strongly associated with objective cognition than a domain-specific assessment of AARC in cognition. Unexpectedly, both perceived social cognitive gains and cognitive losses were associated with poorer cognitive performance. Higher AARC losses in cognition, but not social cognitive gains, were related to greater depression and anxiety, more negative SPA, and poorer SRH suggesting that poorer cognitive functioning may be one of the many variables related to perceptions of losses in cognition. AARC losses in cognition and social cognitive gains respectively showed nonsignificant and negligible associations with engagement in cognitive training.

Our findings support the importance of assessing the coexistence of perceived gains and losses when relating self-perceptions of cognition and/or aging to objective cognitive functioning. However, in contrast to our hypothesis, a global assessment of AARC gains and losses may be more informative of objective cognition compared to a domain-specific assessment of AARC in cognition. This may be due to the global assessment of AARC capturing individuals' perceptions of declines in their mental, physical, and social functioning, alongside cognition, and these are all domains related to objective cognitive functioning (Anstey, 2013). Hence, study results question the previously suggested additional value of domain-specific measures of SPA in predicting matched outcomes (Levy and Leifheit-Limson, 2009).

The higher social cognitive gains reported by those with poorer objective cognition may be due to these individuals making less accurate appraisals of their cognitive performance. This result is in contrast to research showing that cognitively healthy people are generally accurate appraisers of their performance on cognitive tests (Clare et al., 2010). In our study, participants were asked to report perceived cognitive abilities in general, rather than evaluating their performance before and/or after having completed a specific cognitive test; this may explain the difference between our findings and existing literature. However, as we found that more AARC losses in cognition were associated with worse performance on all cognitive tests, this suggests that participants are at least 
Table 4. Path analysis model exploring AARC gains and losses in cognition as predictors of cognition in the three age subgroups while controlling for sex, education, employment status, depression, anxiety, and frequency of cognitive training

\section{PARTICIPANTS AGED 51-65}

STANDARDIZED COEFFICIENT $\beta(95 \% \mathrm{CI}) ; p$-VALUE

PREDICTORS

SELF-ORDERED SEARCH

GRAMMATICAL REASONING

PAIRED ASSOCIATE LEARNING

DIGIT SPAN

AARC gains in cognition

$-.08(-.12,-.05) ;<.001$

$-.08(-.11,-.04) ;<.001$

$-.05(-.09,-.02) ; .004$

$-.04(-.07, .00) ; .052$

$-.05(-.09,-.02) ; .004$

AARC losses in cognition

$-.05(-.08,-.01) ; .013$

$-.09(-.12,-.05) ;<.001$

$-.04(-.08,-.00) ; .038$ RMSEA (90\% CI): .07 (.05, .11); CFI: .99; TLI: .40; SRMR: .01; R R $^{2} 16 \%$

\section{PARTICIPANTS AGED 66-75}

\begin{tabular}{|c|c|c|c|c|}
\hline & SELF-ORDERED SEARCH & GRAMMATICAL REASONING & PAIRED ASSOCIATE LEARNING & DIGIT SPAN \\
\hline AARC gains in cognition & $-.05(-.09,-.02) ; .007$ & $-.13(-.17,-.09) ;<.001$ & $.00(-.04, .04) ; .963$ & $-.04(-.08,-.00) ; .048$ \\
\hline AARC losses in cognition & $-.02(-.06, .02) ; .370$ & $-.10(-.14,-.06) ;<.001$ & $-.09(-.14,-.05) ;<.001$ & $-.07(-.11,-.03) ; .001$ \\
\hline
\end{tabular}

$-.10(-.14,-.06) ;<.001$

$-.09(-.14,-.05) ;<.00$

$-.04(-.08,-.00) ; .048$
$-.07(-.11,-.03) ; .001$

PARTICIPANTS AGED 76 AND OVER

\begin{tabular}{|c|c|c|c|c|}
\hline & SELF-ORDERED SEARCH & GRAMMATICAL REASONING & PAIRED ASSOCIATE LEARNING & DIGIT SPAN \\
\hline AARC gains in cognition & $-.04(-.13, .05) ; .350$ & $-.08(-.17, .01) ; .066$ & $-.00(-.09, .09) ; .946$ & $-.05(-.14, .04) ; .268$ \\
\hline AARC losses in cognition & $.02(-.09, .12) ; .767$ & $-.20(-.30,-.10) ;<.001$ & $-.10(-.20,-.00) ; .049$ & $-.05(-.15, .05) ; .362$ \\
\hline
\end{tabular}

RMSEA = Root mean square error of approximation; CFI = Comparative fit index; TLI = Tucker-Lewis index; SRMR = Standard root mean square residual; $\mathrm{R}^{2}=\mathrm{R}$-squared/coefficient of determination. 
Table 5. Associations of psychological variables and frequency of cognitive training with AARC gains and losses in cognition across three age subgroups

PARTICIPANTS AGED 51-65

\begin{tabular}{|c|c|c|c|c|c|c|}
\hline & \multicolumn{3}{|c|}{ AARC GAINS IN COGNITION } & \multicolumn{3}{|c|}{ AARC LOSSES IN COGNITION } \\
\hline & SIMPLE REGRESSIONS & & MULTIPLE REGRESSION & SIMPLE REGRESSIONS & & MULTIPLE REGRESSION \\
\hline Predictors & $\beta(95 \% \mathrm{CI}) ; p$-value & $\mathrm{R}^{2}$ & $\beta(95 \% \mathrm{CI}) ; p$-value & $\beta(95 \% \mathrm{CI}) ; p$-value & $\mathrm{R}^{2}$ & $\beta(95 \% \mathrm{CI}) ; p$-value \\
\hline Depression & $-.03(-.06, .01) ; .154$ & $.10 \%$ & $-.04(-.08, .01) ; .137$ & $.27(.24, .31) ;<.001$ & $7 \%$ & $.16(.11, .20) ;<.001$ \\
\hline Anxiety & $.01(-.03, .04) ; .653$ & $.01 \%$ & $.04(-.01, .09) ; .078$ & $.23(.20, .26) ;<.001$ & $5 \%$ & $.09(.05, .14) ;<.001$ \\
\hline Attitudes toward own aging & $.02(-.02, .05) ; .296$ & $0 \%$ & $.01(-.03, .04) ; .808$ & $-.15(-.18,-.11) ;<.001$ & $2 \%$ & $-.06(-.09,-.02) ;<.001$ \\
\hline Subjective age & $.07(.03, .10) ;<.001$ & $.10 \%$ & $.06(.02, .10) ; .002$ & $-.21(-.24,-.17) ;<.001$ & $4 \%$ & $-.14(-.18,-.11) ;<.001$ \\
\hline Self-rated health & $.05(.02, .09) ; .004$ & $.03 \%$ & $.03(-.01, .07) ; .137$ & $-.24(-.27,-.20) ;<.001$ & $6 \%$ & $-.12(-.16,-.09) ;<.001$ \\
\hline Frequency of cognitive training & $-.04(-.08,-.01) ; .025$ & $.01 \%$ & & $-.02(-.05, .02) ; .397$ & $0 \%$ & \\
\hline
\end{tabular}

PARTICIPANTS AGED 66-75

AARC GAINS IN COGNITION

AARC LOSSES IN COGNITION

\begin{tabular}{|c|c|c|c|c|c|c|}
\hline & & & & \\
\hline & SIMPLE REGRESSIONS & & MULTIPLE REGRESSION & SIMPLE REGRESSIONS & & MULTIPLE REGRESSION \\
\hline Predictors & $\beta(95 \% \mathrm{CI}) ; p$-value & $\mathrm{R}^{2}$ & $\beta(95 \% \mathrm{CI}) ; p$-value & $\beta(95 \% \mathrm{CI}) ; p$-value & $\mathrm{R}^{2}$ & $\beta(95 \% \mathrm{CI}) ; p$-value \\
\hline Depression & $.00(-.04, .04) ; .897$ & $0 \%$ & $-.02(-.07, .03) ; .498$ & $.28(.24, .31) ;<.001$ & $8 \%$ & $.20(.15, .25) ;<.001$ \\
\hline Anxiety & $.03(-.01, .07) ; .096$ & $.01 \%$ & $.06(.01, .11) ; .022$ & $.21(.18, .25) ;<.001$ & $5 \%$ & $.07(.02, .11) ; .006$ \\
\hline Attitudes toward own aging & $.06(.03, .10) ;<.001$ & $.04 \%$ & $.06(.02, .10) ; .005$ & $-.10(-.14,-.06) ;<.001$ & $1 \%$ & $-.03(-.07, .00) ; .081$ \\
\hline Subjective age & $.11(.07, .15) ;<.001$ & $.10 \%$ & $.11(.07, .15) ;<.001$ & $-.18(-.21,-.14) ;<.001$ & $3 \%$ & $-.12(-.16,-.08) ;<.001$ \\
\hline Self-rated health & $.03(-.01, .07) ; .203$ & $.01 \%$ & $-.01(-.05, .04) ; .743$ & $-.21(-.25,-.17) ;<.001$ & $4 \%$ & $-.11(-.15,-.07) ;<.001$ \\
\hline Frequency of cognitive training & $-.03(-.07, .01) ; .188$ & $.01 \%$ & & $.02(-.02, .06) ; .401$ & $0 \%$ & \\
\hline
\end{tabular}

PARTICIPANTS AGED 76 AND OVER

AARC GAINS IN COGNITION

AARC LOSSES IN COGNITION

\begin{tabular}{|c|c|c|c|c|c|c|}
\hline & & \\
\hline & SIMPLE REGRESSIONS & & MULTIPLE REGRESSION & SIMPLE REGRESSIONS & & MULTIPLE REGRESSION \\
\hline Predictors & $\beta(95 \% \mathrm{CI}) ; p$-value & $\mathrm{R}^{2}$ & $\beta(95 \% \mathrm{CI}) ; p$-value & $\beta(95 \% \mathrm{CI}) ; p$-value & $\mathrm{R}^{2}$ & $\beta(95 \% \mathrm{CI}) ; p$-value \\
\hline Depression & $-.04(-.13, .05) ; .424$ & $.01 \%$ & $-.12(-.22,-.01) ; .034$ & $.33(.25, .41) ;<.001$ & $11 \%$ & $.25(.16, .35) ;<.001$ \\
\hline Anxiety & $.01(-.08, .10) ; .844$ & $0 \%$ & $.06(-.04, .17) ; .227$ & $.32(.24, .40) ;<.001$ & $10 \%$ & $.18(.09, .28) ;<.001$ \\
\hline Attitudes toward own aging & $.03(-.06, .12) ; .465$ & $.01 \%$ & $.04(-.05, .13) ; .429$ & $-.06(-.15, .03) ; .228$ & $.03 \%$ & $-.01(-.09, .07) ; .825$ \\
\hline Subjective age & $.09(.00, .18) ; .051$ & $1 \%$ & $.08(-.01, .17) ; .084$ & $-.19(-.27,-.10) ;<.001$ & $4 \%$ & $-.16(-.24,-.08) ;<.001$ \\
\hline Self-rated health & $-.07(-.16, .02) ; .117$ & $1 \%$ & $-.11(-.20,-.02) ; .019$ & $-.12(-.21,-.03) ; .010$ & $1 \%$ & $-.04(-.12, .05) ; .417$ \\
\hline Frequency of cognitive training & $-.02(-.12, .06) ; .541$ & $.01 \%$ & & $.01(-.08, .10) ; .854$ & $0 \%$ & \\
\hline
\end{tabular}

$\beta=$ Standardized regression coefficient; Partial $\mathrm{R}^{2}=$ Partial R-squared/coefficient of determination. 
somewhat accurate in their perceptions of their cognitive abilities.

Alternatively, the higher social cognitive gains reported by those with poorer objective cognition may be due to these individuals paying more attention to their cognitive gains as this may facilitate acceptance of negative changes and re-establishment of self-efficacy and positive emotional states (Loidl and Leipold, 2019). However, more social cognitive gains were fairly independent from more positive SPA, mental, and physical health. Hence, individuals perceiving more social cognitive gains may not have a general tendency to be more positive in their self-evaluations and may only show this tendency when rating their cognition. Finally, the counterintuitive association of higher social cognitive gains with worse cognitive functioning may be due to the nature of items used to assess AARC in the cognitive domain (Sabatini et al., 2020b). Whereas, the losses items capture perceived decline in cognitive domains, such as memory and processing speed that can be compared to objective performance in cognitive tasks assessing the same domains, the gains items capture social cognition and wisdom, which may not be suitable for comparison with performance on objective cognitive tasks.

This study found that individuals perceiving high cognitive losses may be experiencing poorer cognition across several domains. In line with international evidence on the associations of AARC with mental and physical health (Sabatini et al., 2020a, 2020c), associations of objective cognition with AARC losses were stronger than associations with gains. Among cognitive tests, AARC losses were most strongly associated with GR. This finding was consistent across all age subgroups but strongest in advanced old age; supporting the greater accuracy of older individuals in reporting cognitive difficulties (Jessen et al., 2014). A recent study examining daily within-person variability in AARC and cognitive performance showed that AARC losses predict within-person decreases in inductive reasoning on the same day and decreases from day 1 to the next (Zhu and Neupert, 2020). Despite the methodological differences between this study and ours, both found that among several cognitive domains AARC is most strongly associated with reasoning. This may be due to reasoning being vulnerable to age-related decline (Christensen, 2001).

The small size of the associations of higher AARC losses in cognition with poorer scores on objective cognitive tasks may be due to perceived cognitive losses reflecting individuals' experience of a trajectory of subtle cognitive decline that is not captured with the cross-sectional assessment of objective cognition (Caselli et al., 2014). Research shows that, although SRH generally does not match with objective measures of health, it can be a better predictor of future levels of health than objective measures of health (Idler and Benyamini, 1997). Similarly, AARC losses in cognition may be more strongly associated with objective cognition at the longitudinal level than at the cross-sectional level.

Studies exploring the association of selfperceptions of cognition with objective cognition report mixed results (Burmester et al., 2016; Crumley et al., 2014; Jessen et al., 2014; Jonker et al., 2000). Our results are in line with those studies reporting a statistically significant, but small association between more negative self-perceptions of cognition and poorer objective cognition (Amariglio et al., 2012). This may be due to self-perceptions of cognition being influenced by many psychosocial factors including depressive symptoms and negative ATOA (Segel-Karpas and Palgi, 2019; Siebert et al., 2020). Indeed, in our study, higher levels of AARC losses in cognition are associated with more severe depression and anxiety, more negative ATOA, an older SA, and poorer SRH.

We found that with increasing age depression and anxiety are more strongly associated with AARC losses in cognition, whereas poorer SRH and negative ATOA are most strongly related to AARC losses in middle age. These findings are aligned with literature documenting the co-occurrence of depression, anxiety, poorer cognitive, and physical health in older age (Anstey, 2013; Roehr et al., 2017), but are inconsistent with research supporting the greater self-relevance of ATOA in older age (Kornadt and Rothermund, 2012). The association of more AARC losses in cognition with and older SA is in line with research reporting that those individuals with an older SA pay more attention to age-related losses in memory compared to those who feel their age or younger than their age (Segel-Karpas and Palgi, 2019).

Overall, the small associations of AARC losses in cognition with objective cognition and the small to moderate associations of AARC losses with more negative scores on psychological variables suggest that perceived cognitive losses may be somewhat influenced not only by individuals' objective cognitive ability, but also by their interpretation of the cognitive changes they experience. The way in which older individuals interpret their cognitive changes may be shaped by their beliefs about age-related changes in cognition and their current emotional state (Brothers et al., 2020; Weiss and Kornadt, 2018). Our findings suggest that even though individuals perceiving many cognitive losses may benefit from cognitive interventions (e.g. compensatory cognitive training; Burton et al., 2011), they may benefit more substantially from interventions promoting psychological health (Siebert et al., 2020). 


\section{Strengths and limitations}

This study has several limitations. First, participants were aged 51 and over, so they may not have been old enough to perceive many cognitive losses. Second, analyses are based on a selective group of participants. Indeed, out of the 14,882 participants that took part in the PROTECT annual assessment between January and March 2019, 8826 were excluded from study analyses as they did not complete the AARC questionnaire, the objective cognitive tasks, or they may have had mild cognitive impairment or dementia. Even though there may be a systematic bias in those who did not complete study measures (e.g. less enthusiastic to take part in the study), participants excluded from study analyses had similar demographic profiles and mental and physical health to the study sample.

Third, similarly to most available studies on AARC (Sabatini et al., 2020a), the study sample included a majority of participants who were women, well-educated, and who rated their health as good or excellent, hence extrapolation of results to a broader population should be considered with caution. Fourth, analyses were based on crosssectional data; hence, causality for the associations of perceived cognitive gains and losses with cognitive functioning and psychological variables cannot be inferred. Fifth, even though the AARC questionnaire captures awareness of changes, we explored it in association with current objective cognition instead of cognitive change. Nonetheless, we deemed the AARC questionnaires suitable to assess current self-perceptions of cognition as it is reasonable to assume that current self-perceptions of cognition are on average more positive for those who perceive more social cognitive gains and more negative for those who perceive more AARC losses.

Sixth, as cognitive tests were self-administered online, those participants who are less familiar with technology may perform more poorly compared to when assessed by a researcher. However, in PROTECT, all participants were familiar with the online cognitive tests from previous assessments. As participants level of engagement while undertaking the cognitive tests was not assessed, some critics suggest that it is possible participants could have someone else undertake the cognitive tasks. However, this seems unlikely given the motivation of participants involved in the study. Seventh, even though PROTECT participants are invited to repeat the completion of the cognitive tests in three sessions within a week and then the average score is calculated, numerous participants did not complete the tests over three sessions. In order to optimize the use of data across the cohort, we used only data from the first session. Eighth, the cognitive tests were completed on a separate day (within 2 months) to that on which participants answered the AARC questionnaires. This is a limitation as levels of perceived cognitive gains and losses can vary on a daily basis (Zhu and Neupert, 2020). However, cognitive functioning among individuals without dementia is generally stable over 2 months (e.g. Lövdén et al., 2004). Ninth, items assessing perceived cognitive losses may overlap with symptoms of depression and anxiety (Jessen et al., 2007) and those individuals who are more introspective may score high on perceived cognitive losses, depression, and anxiety (Roberts et al., 2009). Tenth, this study only considered frequency of engagement in computerized online cognitive training available as part of the PROTECT study; this is a limitation as individuals could have been cognitively engaged in many other ways not recorded in this study.

The large sample size and the use of valid measures assessing the coexistence of perceived gains and losses across several domains made it possible to advance knowledge on self-perceptions of cognition by showing that across three age groups more perceived gains and losses both in cognition and across life domains may be associated with poorer cognitive performance.

\section{Conclusions}

This study adds several contributions to existing research. First, when examining the association of SPA with objective cognition, it is important to assess the coexistence of positive and negative SPA across several life domains. Second, both higher levels of perceived social cognitive gains and cognitive losses may be indicative of poorer cognitive functioning, even though associations are either negligible or small and the reasons underlying the association of higher perceived social cognitive gains and poorer cognitive functioning need to be investigated with future research. Third, whereas perceived social cognitive gains are minimally related to psychological variables, AARC losses in cognition are associated with more severe depression and anxiety, more negative ATOA, older SA, and poorer SRH. Overall, poorer cognitive functioning may be only one of the many variables related to AARC losses in cognition.

\section{Conflict of interest}

None. 


\section{Source of funding}

This work was supported by the University of Exeter College of Life and Environmental Sciences (School of Psychology), University of Exeter College of Medicine and Health, and the National Health and Medical Research Council Centre for Research Excellence in Cognitive Health (\#1100579 to Kaarin Anstey).

\section{Description of authors' roles}

SS served as principal investigator of the research, designed the study, conducted data analyses, and took the lead in writing the manuscript. LC contributed to the design of the study, analysis of data, and writing the manuscript. OU contributed to analyses of data, and provided feedback on the draft of the manuscript. AC, $\mathrm{HB}, \mathrm{AH}$, and $\mathrm{CB}$ contributed to data collection and design of the PROTECT study, and provided feedback on the draft of the manuscript. The remaining co-authors provided feedback on the draft of the manuscript.

\section{Acknowledgments}

We are grateful to the University of Exeter for funding a $\mathrm{PhD}$ scholarship for Serena Sabatini to carry out this work. This paper represents independent research funded by the National Institute for Health Research (NIHR) Biomedical Research Centre at South London and Maudsley NHS Foundation Trust and King's College London. Obioha Ukoumunne was supported by the National Institute for Health Research (NIHR) Applied Research Collaboration (ARC) South West Peninsula. The views expressed are those of the author(s) and not necessarily those of the NHS, the NIHR, or the Department of Health and Social Care.

\section{Availability of data and materials}

This study was conducted using secondary data collected as part of the UK version of the PROTECT ongoing study. PROTECT data are available to investigators outside the PROTECT team after request and approval by the PROTECT Steering Committee. Data for the AARC questionnaires will be available from May 2022 .

\section{Supplementary material}

To view supplementary material for this article, please visit https://doi.org/10.1017/S1041610221000375.

\section{References}

Amariglio, R. E. et al. (2012). Subjective cognitive complaints and amyloid burden in cognitively normal older individuals. Neuropsychologia, 50, 2880-2886. https://doi .org/10.1016/j.neuropsychologia.2012.08.011

Anstey, K. J. (2013). Optimizing cognitive development over the life course and preventing cognitive decline: introducing the Cognitive Health Environment Life Course Model (CHELM). International fournal of Behavioral Development, 38, 1-10. https://doi.org/10.1177/0165025413512255

Barrett, A. E. (2003). Socioeconomic status and age identity: the role of dimensions of health in the subjective construction of age. The fournals of Gerontology, Series B: Psychological Sciences and Social Sciences, 58, 101-109. https://doi.org/10.1093/geronb/58.2.S101

Blackburn, D. J., Harkness, K., Reuber, M., Venneri, A., Shanks, M. F. and Wakefield, S. (2014). Memory difficulties are not always a sign of incipient dementia: a review of the possible causes of loss of memory efficiency. British Medical Bulletin, 112, 71-81. https://doi.org/10 $.1093 / \mathrm{bmb} / \mathrm{ldu} 029$

Brothers, A. F., Gabrian, M., Wahl, H.-W. and Diehl, M. K. (2019). A new multidimensional questionnaire to assess awareness of age-related change (AARC). The Gerontologist, 59, e141-e151. https://doi.org/10.1093/ geront/gny006

Brothers, A. F., Kornadt, A. E., Nehrkorn-Bailey, A., Wahl, H.-W. and Diehl, M. K. (2020). The effects of age stereotypes on physical and mental health are mediated by self-perceptions of aging. The fournals of Gerontology, Series B: Psychological Sciences and Social Sciences. https://doi.org/ 10.1093/geronb/gbaa176

Bryant, C., Bei, B., Gilson, K., Komiti, A., Jackson, H. and Judd, F. (2012). The relationship between attitudes to aging and physical and mental health in older adults. International Psychogeriatrics, 24, 1674-1683. https://doi.org/ 10.1017/s 1041610212000774

Burmester, B., Leathem, J. and Merrick, P. (2016). Subjective cognitive complaints and objective cognitive function in aging: a systematic review and meta-analysis of recent cross-sectional findings. Neuropsychology Review, 26, 376-393. https://doi.org/10.1007/s11065-016-9332-2

Burton, C. Z., Vella, L. and Twamley, E. W. (2011). Clinical and cognitive insight in a compensatory cognitive training intervention. American fournal of Psychiatric Rehabilitation, 14, 307-326. https://doi.org/10.1080/ 15487768.2011.622159

Byrne, B. M. (2012). Structural Equation Modeling with Mplus: Basic Concepts, Applications, and Programming. New York, NY: Routledge/Taylor \& Francis Group.

Carstensen, L. L. et al. (2011). Emotional experience improves with age: evidence based on over 10 years of experience sampling. Psychology and Aging, 26, 21-33. https://doi.org/10.1037/a0021285

Caselli, R. J. et al. (2014). Subjective cognitive decline: self and informant comparisons. Alzheimer's \& Dementia, 10, 93-98. https://doi.org/10.1016/j.jalz.2013.01.003

Christensen, H. (2001). What cognitive changes can be expected with normal ageing? Australian and New Zealand Fournal of Psychiatry, 35, 768-775. https://doi.org/10 $.1046 / j .1440-1614.2001 .00966 . x$ 
Clare, L., Whitaker, C. J. and Nelis, S. M. (2010). Appraisal of memory functioning and memory performance in healthy ageing and early-stage Alzheimer's Disease. Aging, Neuropsychology, and Cognition, 17, 462-491. https:// doi.org/10.1080/13825580903581558

Cohen, J. (1988). Statistical Power Analysis for the Behavioral Sciences. Hillsdale, NJ: Lawrence Earlbaum Associates.

Corbett, A. et al. (2015). The effect of an online cognitive training package in healthy older adults: an online randomized controlled trial. Fournal of the American Medical Directors Association, 16, 990-997. https://doi.org/10 .1016/j.jamda.2015.06.014

Crumley, J. J., Stetler, C. A. and Horhota, M. (2014). Examining the relationship between subjective and objective memory performance in older adults: a meta-analysis. Psychology and Aging, 29, 250. https://doi.org/10.1037/ a0035908

Diehl, M. K. and Wahl, H.-W. (2010). Awareness of agerelated change: examination of a (mostly) unexplored concept. The fournals of Gerontology, Series B: Psychological Sciences and Social Sciences, 65B, S340-S350. https://doi.org/ 10.1093/geronb/gbp110

Hertzog, C., Hülür, G., Gerstorf, D. and Pearman, A. (2018). Is subjective memory change in old age based on accurate monitoring of age-related memory change? Evidence from two longitudinal studies. Psychology and Aging, 33, 273-287. https://doi.org/10.1037/pag0000232

Hess, T. M. (2006). Attitudes toward aging and their effects on behavior. In UCLA Center on Aging (Ed.) Handbook of the Psychology of Aging (pp. 379-406). Los Angeles, CA: Elsevier.

Hill, N. L. et al. (2016). Subjective cognitive impairment and affective symptoms: a systematic review. The Gerontologist, 56, e109-e127. https://doi.org/10.1093/geront/gn-w091

Idler, E. L. and Benyamini, Y. (1997). Self-rated health and mortality: a review of twenty-seven community studies. fournal of Health and Social Behavior, 38, 21-37. https://doi .org/10.2307/2955359

Jessen, F. et al. (2014). A conceptual framework for research on subjective cognitive decline in preclinical Alzheimer's disease. Alzheimer's $\mathcal{E}$ Dementia, 10, 844-852. https://doi .org/10.1016/j.jalz.2014.01.001

Jessen, F. et al. (2007). Patterns of subjective memory impairment in the elderly: association with memory performance. Psychological Medicine, 1753-1762. https:// doi.org/10.1017/S0033291707001122

Jonker, C., Geerlings, M. I. and Schmand, B. A. (2000). Are memory complaints predictive for dementia? A review of clinical and population-based studies. International fournal of Geriatric Psychiatry, 15, 983-991. https://doi.org/10.1002/ 1099-1166(200011)15:11

Jorm, A. F. and Jacomb, P. A. (1989). The Informant Questionnaire on Cognitive Decline in the Elderly (IQCODE): socio-demographic correlates, reliability, validity and some norms. Psychological Medicine, 19, 10151022. https://doi.org/10.1017/S0033291700005742

Kaspar, R., Gabrian, M., Brothers, A. F., Wahl, H.-W. and Diehl, M. K. (2019). Measuring awareness of agerelated change: development of a 10-Item short form for use in large-scale surveys. The Gerontologist, 59, e130-e140. https://doi.org/10.1093/geront/gnx213
Kornadt, A. E. and Rothermund, K. (2012).

Internalization of age stereotypes into the self-concept via future self-views: a general model and domain-specific differences. Psychology and Aging, 27, 164-172. https://doi .org/10.1037/a0025110

Kroenke, K., Spitzer, R. L. and Williams, J. B. (2001). The PHQ-9: validity of a brief depression severity measure. Fournal of General Internal Medicine, 16, 606-613. https:// doi.org/10.1046/j.1525-1497.2001.016009606.x

Lawton, M. P. (1975). The Philadelphia geriatric center morale scale: a revision. Fournal of Gerontology, 30, 85-89.

Levy, B. R. and Leifheit-Limson, E. (2009). The stereotype-matching effect: greater influence on functioning when age stereotypes correspond to outcomes. Psychology and Aging, 24, 230-233. https://doi.org/10.1037/a0014563

Loid1, B. and Leipold, B. (2019). Facets of accommodative coping in adulthood. Psychology and Aging, 34, 640-654. https://doi.org/10.1037/pag0000378

Lövdén, M., Rönnlund, M., Wahlin, Å., Bäckman, L., Nyberg, L. and Nilsson, L.-G. (2004). The extent of stability and change in episodic and semantic memory in old age: demographic predictors of level and change. The fournals of Gerontology, Series B: Psychological Sciences and Social Sciences, 59, P130-P134. https://doi.org/10.1093/ geronb/59.3.P130

Meisner, B. A. (2012). A meta-analysis of positive and negative age stereotype priming effects on behavior among older adults. The fournals of Gerontology, Series B: Psychological Sciences and Social Sciences, 67, 13-17. https:// doi.org/10.1093/geronb/gbr062

Miche, M., Wahl, H.-W., Diehl, M. K., Oswald, F., Kaspar, R. and Kolb, M. (2014). Natural occurrence of subjective aging experiences in community-dwelling older adults. The fournals of Gerontology, Series B: Psychological Sciences and Social Sciences, 69, 174-187. https://doi.org/10 $.1093 /$ geronb/gbs 164

Owen, A. M. et al. (2010). Putting brain training to the test. Nature, 465, 775-778. https://doi.org/10.1038/nature/09042

Palsson, S. P., Ostling, S. and Skoog, I. (2001). The incidence of first-onset depression in a population followed from the age of 70 to 85. Psychological Medicine, 31, 11591168. https://doi.org/10.1017/S0033291701004524

Rabin, L. A. et al. (2015). Subjective cognitive decline in older adults: an overview of self-report measures used across 19 international research studies. Fournal of Alzheimer's Disease, 48, S63-S86. https://doi.org/10.3233/JAD-150154

Roberts, J. L., Clare, L. and Woods, R. T. (2009). Subjective memory complaints and awareness of memory functioning in mild cognitive impairment: a systematic review. Dementia and Geriatric Cognitive Disorders, 28, 95109. https://doi.org/10.1159/000234911

Roehr, S. et al. (2017). Subjective cognitive decline is longitudinally associated with lower health-related quality of life. International Psychogeriatrics, 29, 1939-1950. https:// doi.org/10.1017/S1041610217001399

Sabatini, S. et al. (2020a). Associations of awareness of agerelated change with emotional and physical well-being: a systematic review and meta-analysis. The Gerontologist, 60, e477-e490. https://doi.org/10.1093/geront/gnz101

Sabatini, S. et al. (2020b). International relevance of two measures of awareness of age-related change (AARC). BMC 
Geriatrics, 20, 359. https://doi.org/10.1186/s12877-02001767-6

Sabatini, S. et al. (2020c). The cross-sectional relationship between pain and awareness of age-related changes. British fournal of Pain. https://doi.org/10.1177/ 2049463720961798

Segel-Karpas, D. and Palgi, Y. (2019). 'It is nothing more than a senior moment': the moderating role of subjective age in the effect of change in memory on self-rated memory. Aging E Mental Health, 23, 272-276. https://doi.org/10 $.1080 / 13607863.2017 .1399350$

Seidler, A. L. and Wolff, J. K. (2017). Bidirectional associations between self-perceptions of aging and processing speed across 3 years. The fournal of Gerontopsychology and Geriatric Psychiatry, 30, 49-59. https://doi.org/10.1024/1662-9647/a000165

Siebert, J. S., Braun, T. and Wahl, H. W. (2020). Change in attitudes toward aging: cognitive complaints matter more than objective performance. Psychology and Aging, 35, 357-368. https://doi.org/10.1037/pag0000451

Siebert, J. S., Wahı, H.-W., Degen, C. and Schröder, J. (2018). Attitude toward own aging as a risk factor for cognitive disorder in old age: 12-year evidence from the ILSE study. Psychology and Aging, 33, 461-472. https://doi .org/10.1037/pag0000252

Slot, R. E. R. et al. (2018). Subjective Cognitive Impairment Cohort (SCIENCe): study design and first results. Alzheimer's Research E Therapy, 10, 1-13. https://doi.org/ 10.1186/s13195-018-0390-y

Smart, C. M. and Krawitz, A. (2015). The impact of subjective cognitive decline on Iowa Gambling Task performance. Neuropsychology, 29, 971. https://doi.org/10 $.1037 /$ neu0000204

Spitzer, R. L., Kroenke, K., Williams, J. B. and Lowe, B. (2006). A brief measure for assessing generalized anxiety disorder: the GAD-7. Archives of Internal Medicine, 166, 1092-1097. https://doi.org/10.1001/archinte.166.10.1092

Steverink, N., Westerhof, G. J., Bode, C. and DittmannKohli, F. (2001). The personal experience of aging, individual resources, and subjective well-being. The fournals of Gerontology, Series B: Psychological Sciences and Social Sciences, 56, 364-373. https://doi.org/10.1093/ geronb/56.6.P364
Sullivan, K. W., Law, W. A., Loyola, L., Knoll, M. A., Shub, D. E. and French, L. M. (2020). A novel intervention platform for service members with subjective cognitive complaints: implementation, patient participation, and satisfaction. Military medicine, 185, 326-333. https:// doi.org/10.1093/milmed/usz218

Tandetnik, C. et al. (2015). Ascertaining subjective cognitive decline: a comparison of approaches and evidence for using an age-anchored reference group. Fournal of Alzheimer's Disease, 48, S43-S55. https://doi.org/10.3233/ JAD-150251

Wahl, H.-W., Konieczny, C. and Diehl, M. K. (2013). Experiencing age-related change in adulthood: an exploratory study based on the concept of "Awareness of Age-Related Change" (AARC). Zeitschrift für Entwicklungspsychologie und Pädagogische Psychologie, 45, 66-76. https://doi.org/10.1026/0049-8637/a000081

Wang, L. et al. (2004). Subjective memory deterioration and future dementia in people aged 65 and older. Fournal of the American Geriatrics Society, 52, 2045-2051. https://doi.org/ 10.1111/j.1532-5415.2004.52568.x

Ware, J. E. J. and Sherbourne, C. D. (1992). The MOS 36-item short-form health survey (SF-36). Conceptual framework and item selection. Medical Care, 30, 473-483. https://doi.org/10.1177/016327879702000102

Weiss, D. and Kornadt, A. E. (2018). Age-stereotype internalization and dissociation: contradictory processes or two sides of the same coin? Current Directions in Psychological Science, 27, 477-483. https://doi.org/10.1177/ 0963721418777743

Weyerer, S. et al. (2013). Incidence and predictors of depression in non-demented primary care attenders aged 75 years and older: results from a 3-year follow-up study. Age and Ageing, 42, 173-180. https://doi.org/10.1093/ageing/ afs 184

World Health Organization (2020). Dementia. https:// www.who.int/news-room/fact-sheets/detail/dementia

Zhu, X. and Neupert, S. D. (2020). Dynamic awareness of age-related losses predict concurrent and subsequent changes in daily inductive reasoning performance. British fournal of Developmental Psychology. https://doi.org/10.1111/ bjdp. 12344 